\title{
Article \\ Interfacial Pockels Effect of Solvents with a Larger Static Dielectric Constant than Water and an Ionic Liquid on the Surface of a Transparent Oxide Electrode
}

\author{
Akihiro Okada ${ }^{1}$, Takayoshi Kobayashi ${ }^{2,3,4}$ and Eiji Tokunaga ${ }^{1,4, *}$ \\ 1 Department of Physics, Faculty of Science, Tokyo University of Science, 1-3 Kagurazaka, Shinjuku-ku, \\ Tokyo 162-8601, Japan; 1220511@ed.tus.ac.jp \\ 2 Advanced Ultrafast Laser Research Center, Department of Engineering Science, The University of \\ Electro-Communications, Chofu, Tokyo 182-8585, Japan; kobayashi1901@gmail.com \\ 3 Department of Electrophysics, National Chiao Tung University, 1001 Ta Hsueh Road, Hsinchu 300, Taiwan \\ 4 Research Center for Water Frontier Science and Technology, Tokyo University of Science, 1-3 Kagurazaka, \\ Shinjuku-ku, Tokyo 162-8601, Japan \\ * Correspondence: eiji@rs.tus.ac.jp
}

Citation: Okada, A.; Kobayashi, T.; Tokunaga, E. Interfacial Pockels Effect of Solvents with a Larger Static Dielectric Constant Than Water and an Ionic Liquid on the Surface of a Transparent Oxide Electrode. Appl. Sci. 2022, 12, 2454. https://doi.org/ 10.3390/app12052454

Academic Editor: Oriele Palumbo

Received: 4 February 2022

Accepted: 23 February 2022

Published: 26 February 2022

Publisher's Note: MDPI stays neutral with regard to jurisdictional claims in published maps and institutional affiliations.

Copyright: (C) 2022 by the authors. Licensee MDPI, Basel, Switzerland. This article is an open access article distributed under the terms and conditions of the Creative Commons Attribution (CC BY) license (https:// creativecommons.org/licenses/by/ $4.0 /)$.
Featured Application: Electro-optic modulators or interfacial sensors involving water, organic solvents, and ionic liquids.

\begin{abstract}
The optical Pockels effect is a change in the refractive index proportional to an applied electric field. As a typical example of the interfacial Pockels effect occurring at interfaces where the spatial inversion symmetry is broken, it is known that water in the electric double layer (EDL) on the transparent oxide electrode surface has a large Pockels coefficient, but the physical factors that determine its size are not clear. Therefore, we experimentally studied the Pockels effect of water and other characteristic liquids - formamide (FA), methylformamide (NMF) (these two have larger static dielectric constants than water), dimethylformamide (DMF), and an ionic liquid that is itself salts (IL, [BMIM] $\left[\mathrm{BF}_{4}\right]$ ) - and evaluated their Pockels coefficients in the EDL on the transparent electrode surface. The magnitude of the Pockels coefficient was found to be in the order of water, DMF, FA, NMF, and IL, with the magnitude of the static dielectric constant not being an important factor.
\end{abstract}

Keywords: interfacial Pockels effect; electric double layer; water; formamide; methylformamide; dimethylformamide; ionic liquid; electro-modulation spectroscopy; indium tin oxide; multichannel lock-in amplifier

\section{Introduction}

When a voltage is applied between electrodes immersed in a solution, charged particles in the solution redistribute to cancel out the potential difference in the bulk solution, forming an electric double layer (EDL) with a concentrated electric field at the electrodesolution interface [1,2]. The EDL at the electrode interface has traditionally been studied as a stage for electrochemical reactions, but in recent years it has been widely used for EDL capacitors [3], EDL transistors [4,5], electric field-induced ferromagnetism [6], and electric field-induced superconductivity [7]. Ionic liquids have been attracting attention as a new material suitable for these applications. Therefore, researches to obtain a fundamental understanding of the properties of the EDL of ionic liquids has been actively conducted both experimentally and theoretically [8-11].

The electrode interface is also an interesting object of study from the viewpoint of nonlinear optics because of its broken spatial inversion symmetry. In other words, secondorder nonlinear optical effects, such as sum frequency generation and Pockels effect, appear. The Pockels effect at the solid-liquid interface was first observed in the EDL of water at the interface of transparent oxide electrodes [12], and was found to have a Pockels coefficient 
an order of magnitude larger [13] than that of the electro-optic crystal $\mathrm{LiNbO}_{3}[14,15]$, which has been put to practical use. In a series of subsequent studies, it was reported that the Pockels effect at the solid-liquid interface is relatively large even in hydrogenbonding solvents, such as alcohols [16], that it is small in water at the interface of noble metal electrodes, such as platinum and silver $[17,18]$, and that an optical modulator with a practical level of signal magnitude is possible using the Pockels effect in water at the transparent electrode interface $[19,20]$. In the observation of the Pockels effect, an electrolyte is dissolved in the solvent to make it conductive and form an EDL, and it is known that the effect does not depend on the type of electrolyte ion [12,16].

Among the liquid-electrode combinations investigated so far [13,16-18,21], the Pockels coefficient of water at the transparent oxide electrode interface is reported to be the largest $(\sim 200 \mathrm{pm} / \mathrm{V})$ to date. However, it is unclear why this combination exhibits this value, i.e., what are the physical factors that determine the magnitude and sign of the Pockels coefficient of the liquid at the electrode interface, and it is desirable to elucidate the physical mechanism that can predict the Pockels coefficient at any given interface. If this can be clarified, it will be possible to develop optical modulators and interfacial sensors using the interfacial Pockels effect that is ubiquitous at all interfaces. The factors suggested so far are that the hydrogen bonding network of the solvent and the interaction of the solvent molecules with the oxide or polar surface may be important, since the coefficient is relatively large for hydrogen bonding solvents and small for noble metal interfaces [16-18].

In this paper, we present an experimental study to measure the Pockels coefficient at the transparent electrode interface of formamides [22,23] (formamide (FA), $N$-methylformamide (NMF), dimethylformamide (DMF), the first two of which are solvents with a larger static dielectric constant than water), and an ionic liquid (IL) ([BMIM] $\left[\mathrm{BF}_{4}\right]: 1$-butyl-3methylimidazolium tetrafluoroborate) [24,25], which is a unique liquid consisting only of electrolyte ions, was experimentally studied to determine the Pockels coefficient at the transparent electrode interface. One of the main features that distinguish water from other solvents is the strong hydrogen bonding and the large static dielectric constant $[26,27]$. Since formamide and methylformamide have larger dielectric constants than water, if a large Pockels coefficient is obtained, it would indicate that dielectric constant is an important factor. For comparison, we also included dimethylformamide, which does not have a large dielectric constant but is a derivative of FA or NMF, and measured them in comparison with water. As for ionic liquids, there is a recent report suggesting the Pockels effect of ionic liquids [28], but the magnitude of the coefficient has not been evaluated. Figure 1 shows the molecular structures of the solvents studied and Table 1 summarizes the physical properties of each solvent together with methanol $(\mathrm{MeOH})$, ethanol $(\mathrm{EtOH})$, and dimethyl sulfoxide (DMSO).

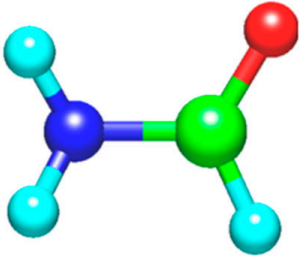

FA

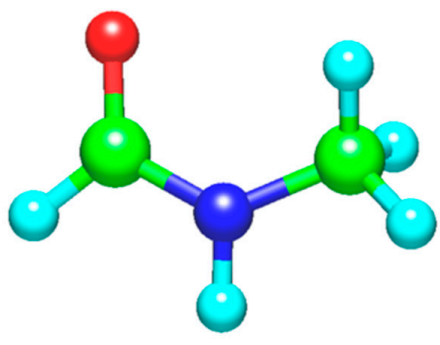

NMF

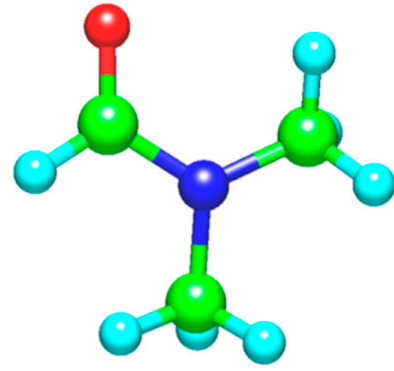

DMF

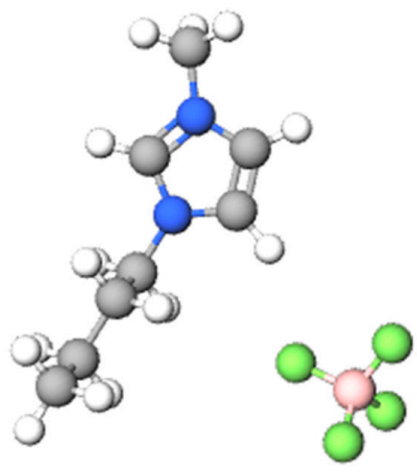

$[\mathrm{BMIM}]\left[\mathrm{BF}_{4}\right]$

Figure 1. The molecular structures of the solvents studied: formamide $\left(\mathrm{FA}, \mathrm{HCONH}_{2}\right), \mathrm{N}$ methylformamide ( $\mathrm{NMF}, \mathrm{CH}_{3} \mathrm{NHCHO}$ ), dimethylformamide ( $\mathrm{DMF},\left(\mathrm{CH}_{3}\right)_{2} \mathrm{NCHO}$ ), and 1-butyl-3methylimidazolium tetrafluoroborate ([BMIM] $\left[\mathrm{BF}_{4}\right], \mathrm{C}_{8} \mathrm{H}_{15} \mathrm{BF}_{4} \mathrm{~N}_{2}$ ). 
Table 1. Physical properties of solvents: values at $20^{\circ} \mathrm{C}$ for water, $\mathrm{MeOH}, \mathrm{EtOH}, \mathrm{DMSO}$ [16].

\begin{tabular}{|c|c|c|c|c|c|c|c|c|}
\hline & $\begin{array}{c}\text { Static } \\
\text { Dielectric } \\
\text { Constant }\end{array}$ & $\begin{array}{c}\text { Dipole } \\
\text { Moment [D] }\end{array}$ & Density $\left[\mathrm{g} / \mathrm{cm}^{3}\right]$ & Viscosity $[\mathrm{mPa} \cdot \mathrm{s}]$ & Boiling Point $\left[{ }^{\circ} \mathrm{C}\right]$ & Melting Point $\left[{ }^{\circ} \mathrm{C}\right]$ & $\begin{array}{l}\text { Refractive } \\
\text { Index }\end{array}$ & $\begin{array}{c}\text { Molecular } \\
\text { Weight }\end{array}$ \\
\hline water & 80.2 & 1.85 & 0.998 & 1.00 & 100 & 0 & 1.333 & 18.015 \\
\hline $\mathrm{MeOH}$ & 33.0 & 1.66 & 0.792 & 0.59 & 64.7 & -97.6 & 1.329 & 32.05 \\
\hline $\mathrm{EtOH}$ & 25.3 & 1.44 & 0.789 & 1.2 & 78.37 & -114.1 & 1.361 & 46.07 \\
\hline FA & $109.5[22,23]$ & 3.37 & 1.133 & $3.23\left(25^{\circ} \mathrm{C}\right)$ & 210 & $2-3$ & 1.447 & 45.04 \\
\hline NMF & $186.0[22,23]$ & 3.83 & 1.011 & $1.72\left(25^{\circ} \mathrm{C}\right)$ & $198 \sim 199$ & -4 & 1.43 & 59.07 \\
\hline DMF & $37.4[22,23]$ & 3.82 & 0.944 & $0.71\left(25^{\circ} \mathrm{C}\right)$ & 153 & -61 & 1.43 & 73.09 \\
\hline DMSO & 47.2 & 3.96 & 1.10 & 1.99 & 189 & 19 & 1.479 & 78.14 \\
\hline $\begin{array}{c}{[\mathrm{BMIM}]} \\
{\left[\mathrm{BF}_{4}\right]}\end{array}$ & 13.9 [29] & & $1.21\left(20^{\circ} \mathrm{C}\right)$ & $132\left(20^{\circ} \mathrm{C}\right)[25]$ & & & 1.41 & 226.02 \\
\hline
\end{tabular}

\section{Materials and Methods}

In the experiment, we measured the change in the transmitted light intensity proportional to the electric field applied to the solution $(\Delta T)$ and the magnitude of the electric field actually felt by the solution $(E)$. Then, based on these data, we estimated the refractive index change of each layer by simulation using the transfer matrix method and determined the Pockels coefficient, following the procedure in Refs. $[12,13,16]$.

For sample solutions, electrolytes were added to make a $0.1 \mathrm{M}$ solution in $10 \mathrm{~mL}$ of each solvent. Deionized water was used as a $0.1 \mathrm{M} \mathrm{NaCl}$ aqueous solution and FA (98.5+\%, Fujifilm Wako, Osaka, Japan), NMF (99.0+\%, Fujifilm Wako, Osaka ,Japan), and DMF $(99.5+\%$, Fujifilm Wako, Osaka ,Japan) were used without further purification as $0.1 \mathrm{M} \mathrm{LiCl}$ solutions. [BMIM] $\left[\mathrm{BF}_{4}\right]$ ( $\geq 98 \%$, Merck, Darmstadt, Germany) was used as it was without any dissolved electrolyte because it is an electrolyte itself. [BMIM][BF $]$ was selected because it is a commonly used ionic liquid that is relatively inexpensive to obtain and its physical properties show typical values for ionic liquids. An open rectangular glass or quartz cell of a size appropriate for the following light transmission measurement was about $80 \%$ filled with each solution.

The experimental setup for electro-modulation spectroscopy is shown in Figure 2. First, we measured the change in the transmission spectrum $(\Delta T)$ due to the application of an external electric field. For this purpose, two $12 \times 50 \mathrm{~mm}$ ITO transparent electrodes (Geomatec, Yokohama, Japan) with a $300 \pm 20 \mathrm{~nm}$ thick indium tin oxide (ITO) film (carrier density $1 \times 10^{21} \mathrm{~cm}^{-3}$ resistivity $1.2 \times 10^{-4} \Omega / \mathrm{cm}$ ) on a $1.1 \mathrm{~mm}$ thick glass substrate were prepared. The two electrode plates were immersed in each solution, facing each other with the conductive side facing inward. The electrode plates were immersed up to $25 \mathrm{~mm}$ in the lower half. Collimated white light from a laser-driven light source (LDLS Xe lamp, EQ-99X, Energetiq, Wilmington, MA, USA) was used as the probe light, and its beam diameter was narrowed down to about $8 \mathrm{~mm}$ with an iris to be transmitted only through one electrode. An alternating current (AC) voltage was applied to this electrode and the other electrode was grounded. In all experiments, the cells were set up in such a way that light was transmitted from the solution to the ITO thin film and then to the glass substrate in that order. The light transmitted through the electrode, and through the solution and glass cell, was spectrally dispersed into 128 wavelengths with a spectrometer and sent through a bundled fiber array to 128 avalanche photodiodes (APDs, S5343, Hamamatsu Photonics, Hamamatsu, Japan), respectively. The photocurrent detected with each APD was converted to a voltage by a transimpedance preamplifier and sent to a 128-channel lock-in amplifier (MLA, 7210, Signal Recovery, Edinburgh, UK). The transmitted intensity change spectrum $(\Delta T)$ synchronized with the AC voltage of frequency $f$ applied between the electrodes was detected simultaneously at 128 wavelengths. The spectrometer was used with a diffraction grating with 150 lines $/ \mathrm{mm}$ and a blaze wavelength of $500 \mathrm{~nm}$. In order to measure water and [BMIM] $\left[\mathrm{BF}_{4}\right]$ in the ultraviolet region (shorter than $350 \mathrm{~nm}$ ), which is absorbed by glass, the glass cell and all the lenses were replaced with those made of quartz, and the grating was changed to that with 150 lines/mm and $300 \mathrm{~nm}$ blaze. An AC voltage of $210 \mathrm{~Hz}$ with $1 \mathrm{~V}$ amplitude was applied to all the solutions. All the experiments were performed at room-temperature $\left(20^{\circ} \mathrm{C}\right)$. Since $\Delta T$ reflects the spectral intensity of the 
white light source and the spectral sensitivity of the measurement system, the experimental results were plotted as $\Delta T / T$, normalized by the transmitted light intensity of the probe light, $T$, which was also measured with the above multi-lock-in measurement system by chopper modulation.

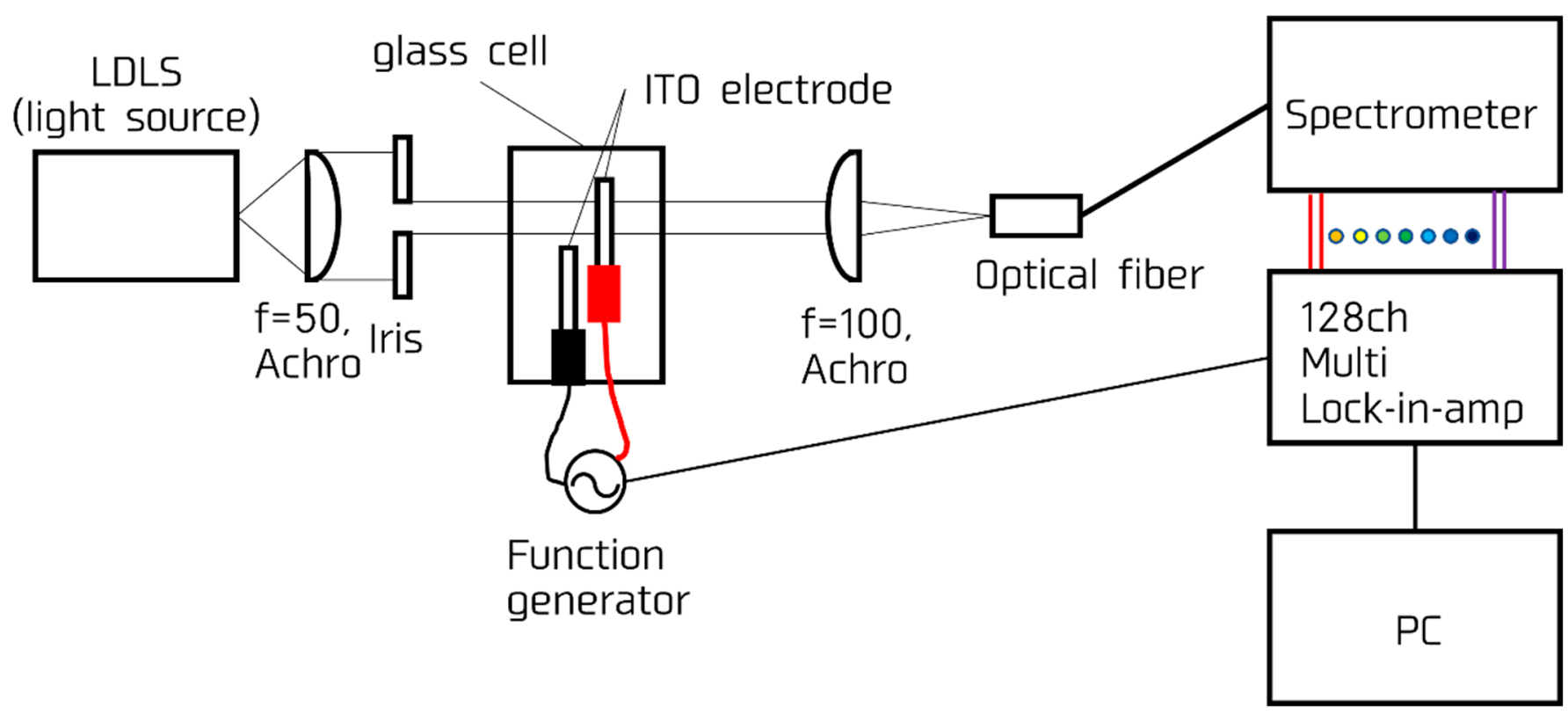

Figure 2. Experimental setup for electro-modulation spectroscopy.

Next, the AC impedance method was used to measure the voltage actually applied to the electrode interface. The AC impedance method was performed by using two identical ITO electrodes as two terminals by a two-terminal method, using a potentiometer/galvanostat SI1287 (Solartron, Victorville, CA, USA) and an impedance analyzer SI1260 (Solartron, Victorville, CA, USA). The electrode onto which the light was irradiated used as the working electrode, and the other electrode was used as the counter electrode. The voltage applied during the measurement was $50 \mathrm{mV} \mathrm{AC}$, and the measurement frequency range was $10^{-1}-10^{7} \mathrm{~Hz}$. However, the data in the high-frequency region is cut off in Figure A1 in Appendix A because it fluctuates significantly.

\section{Results}

The change in the normalized transmission spectrum $(\Delta T / T)$ for each solvent is shown by the black line in Figure 3. We can see that each spectrum has a shape similar to those previously observed $[13,16]$. The result of the impedance measurement of each solution is shown by the black line in Figure A1 in Appendix A. Each graph on the left is a Cole-Cole plot, which is a plot of impedance in the complex plane. The two graphs on the right are bode plots, where the top shows the magnitude of the impedance, and the bottom shows the phase. The results of fitting with parameters in the equivalent circuit assumed (shown later) are summarized in Table A1 and the fitting curves (red) in Figure A1. 

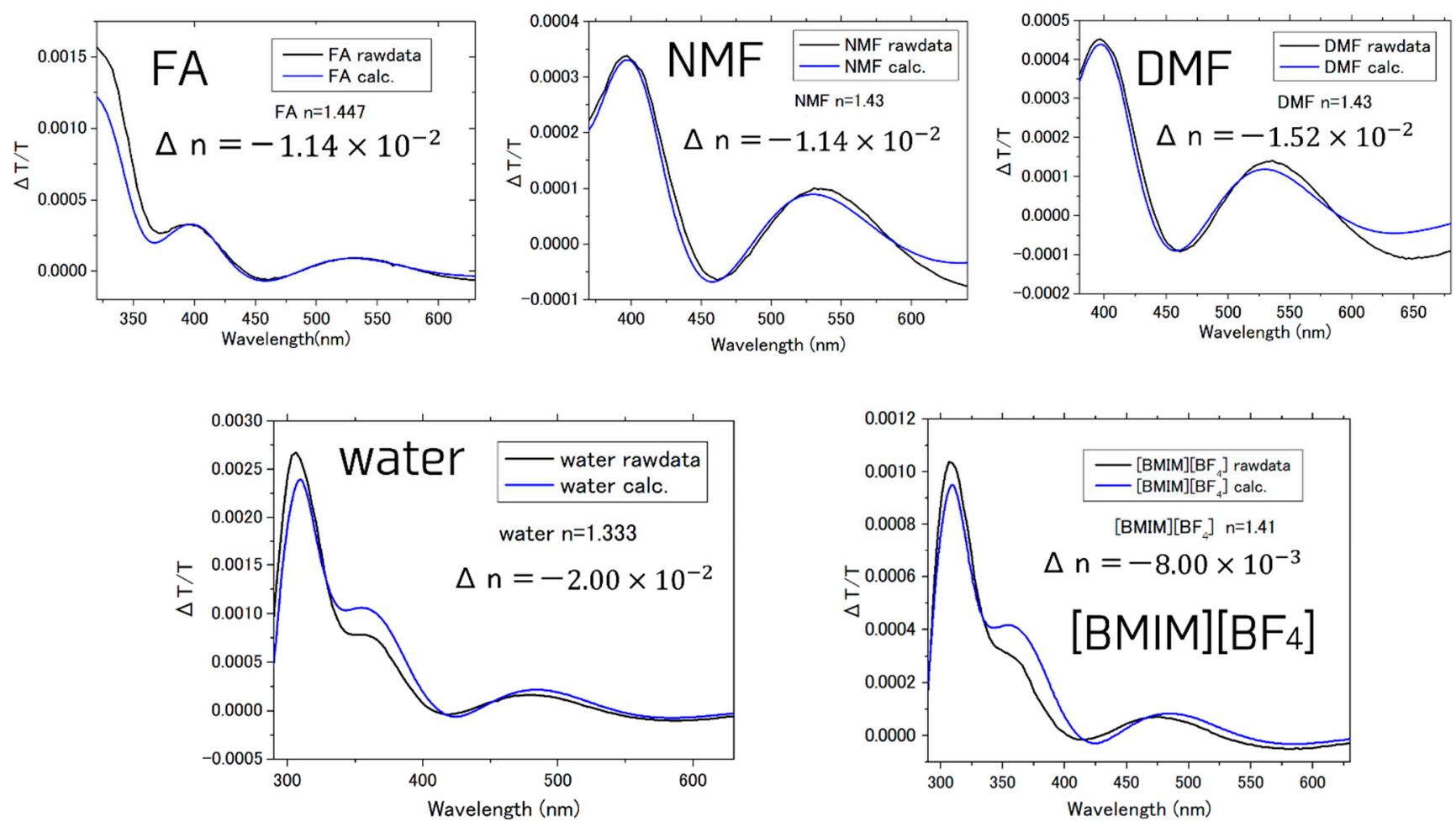

Figure 3. Experimental $\Delta T / T$ spectra of FA, NMF, DMF, water, and IL (black lines) and calculated $\Delta T / T$ spectra of them assuming $\Delta n$ within the EDL of the liquid. The sign of the refractive index change is defined as negative because the refractive index change at the electrode interface is negative when positive voltage is applied.

\subsection{Analysis Methods}

3.1.1. Estimation of $\Delta n$ from Electro-Modulation Spectra Simulated by Transfer Matrix Method

To reproduce the experimental $\Delta T / T$ spectra, the calculations were performed with a self-made Visual Basic program using the transfer matrix method [30], assuming a change in the refractive index of each solvent $\Delta n$ on the electrode, to obtain the results shown by the blue lines in Figure 3. By application of the voltage, the EDL (EDL) is formed on the surface of the ITO electrode where the charge is accumulated, and the refractive index is considered to change in this layer compared to the bulk. At the same time as the EDL is formed, the refractive index change due to the shift in the effective band gap energy [31] caused by the band-population effect $[32,33]$ of the n-type degenerate semiconductor also occurs on the ITO surface induced by the strong electric field at the interface. This region is called the space charge layer (SCL) $[12,13,16]$. As shown in Figure 4, therefore, firstly we calculated the transmittance before a voltage is applied, $T$, through a single layer structure with a bulk ITO film sandwiched between a semi-infinite solution and a semi-infinite glass. Secondly, we calculated the transmittance $T^{\prime}$ when the voltage is applied, through a threelayer structure in which light is incident from the semi-infinite bulk solution, transmitted through the EDL of the solution, the SCL of ITO, and the bulk ITO film, and exits onto the semi-infinite glass substrate. As a result, the calculated $\Delta T / T$ spectra were obtained with $\Delta T=T^{\prime}-T$. In the actual experiment, the probe light entered the solution again after transmitted through the glass substrate, but this part can be ignored because we only consider the difference before and after the refractive index change. In reality, the refractive index change in the EDL and SCL is spatially distributed, but we assume that the layers have effective thickness with spatially uniform refractive index change as before $[12,13,16]$. 
Before voltage applied

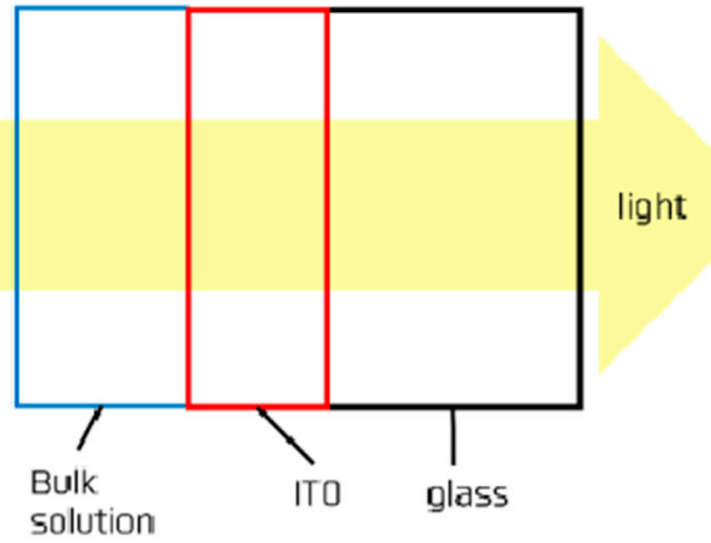

After voltage applied

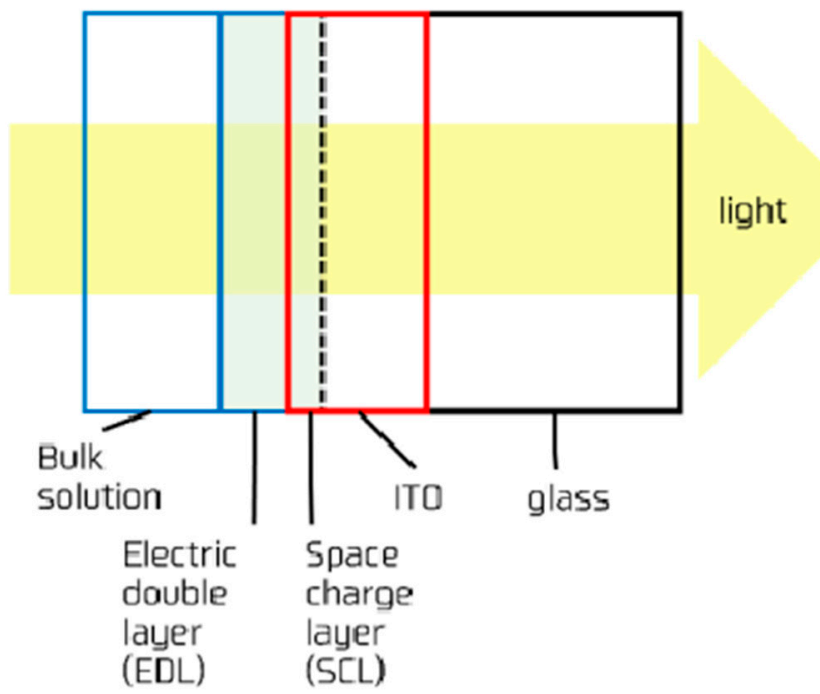

Figure 4. The models of the samples for calculating $\Delta T / T=\left(T^{\prime}-T\right) / T$. Left: The single layer model (bulk ITO) without applied voltage for calculating $T$. Right: The three layer model (EDL, SCL, bulk ITO) with applied voltage for calculating $T^{\prime}$.

In order to determine the $\Delta n$ that occurs in the EDL, we have to exclude the refractive index change $\Delta n_{\text {SCL }}$ that occurs in the SCL. This was handled as follows. The refractive index change of solution EDL has almost no wavelength dependence in the range of 300 to $650 \mathrm{~nm}$, reflecting the transparency of the solution, while that of the SCL in ITO has a wavelength dependence because it is caused by the net absorption change at the band edge due to the blue/red shift in the effective band gap upon positive/negative voltage application (as proved by the transmittance increase around $310 \mathrm{~nm}$ in Figure 3) and, thus, the refractive index change has the wavelength dependence associated with the absorption change through the Kramers-Kronig $(\mathrm{KK})$ relations. Therefore, we determined the complex refractive index $\Delta n_{\mathrm{SCL}}+i \Delta \kappa_{\mathrm{SCL}}\left(\kappa_{\mathrm{SCL}}\right.$ : extinction coefficient) calculated from the complex permittivity assuming a multi-oscillator Lorentz model, so that $\Delta \kappa_{\mathrm{SCL}}$ reproduces the spectrum of $\Delta T / T$ around $310 \mathrm{~nm}$ (net absorption decrease, $\Delta \kappa_{\mathrm{SCL}}<0$ ). In this way, we uniquely determined the $\Delta n_{\mathrm{SCL}}$ in the visible region that satisfies the KK relations with $\Delta \kappa_{\mathrm{SCL}}$. The $\Delta T / T$ in the visible region cannot be reproduced by this $\Delta n_{\mathrm{SCL}}$ alone, but it can only be reproduced by adding $\Delta n$ without wavelength dependence in the EDL. As a result, $\Delta n$ was uniquely determined. For a detailed explanation of this part, please refer to Refs. [12,13,16].

Each $\Delta n$ assumed in the calculation is shown in Figure 3. Here, all $\Delta n$ values are based on the assumption that the EDL thickness is $2 \mathrm{~nm}$ (from the Debye-Hückel length scale of $0.1 \mathrm{M}$ electrolyte ionic strength, although the Debye length of ionic liquids is much shorter). This is different from the actual thickness of the EDL under an AC voltage of $210 \mathrm{~Hz}$. Therefore, the $\Delta n$ displayed is an indication of the relative magnitude of the signal between the solutions, but the absolute magnitude itself is not quantitatively correct. However, since the intra-layer electric field is also calculated from the intra-layer voltage using the same EDL thickness of $2 \mathrm{~nm}$, the magnitude of the Pockels coefficient does not directly depend on the assumed layer thickness, so that the magnitude of the Pockels coefficient is quantitatively justified. That is, taking the thickness of the EDL as $d$ and the appropriate proportionality constant as $C, \Delta T / T=C \Delta n d$ and the Pockels coefficient $=\Delta n / E$. Then, the Pockels coefficient is independent of $d$ since $\Delta n=(\Delta T / T) /(C d)$ and the electric field $E=V / d[13]$.

For the calculation of $T$, the same complex refractive index of ITO as in Ref. [16], 1.52 for the refractive index of the glass, and the values in Table 1 for those of the solvents were 
used. In the SCL, it is assumed that $\Delta n_{\mathrm{SCL}}+i \Delta \kappa_{\mathrm{SCL}}$ has the same wavelength dependence as in Refs. [13,16], $\Delta n_{\mathrm{SCL}}=-0.00042$ at $500 \mathrm{~nm}$ for water with the SCL thickness being $30 \mathrm{~nm}[13,16]$, and $\left|\Delta n_{\mathrm{SCL}}\right|$ is reduced in the other solvents so that $\Delta n_{\mathrm{SCL}} / \Delta n$ is the same. The thickness of ITO (commercially specified as $300 \pm 20 \mathrm{~nm}$ ), which determines the interference fringe patterns observed in $\Delta T / T$, is taken to be $320 \mathrm{~nm}$ for FA, NMF, DMF and $285 \mathrm{~nm}$ for water and IL for the best fit.

\subsubsection{Estimation of Electric Field in Electric Double Layer by Equivalent Circuit and} Evaluation of Pockels Coefficient

Next, let us consider the electric field in each layer from the results of the impedance measurement. We applied the equivalent circuit in Figure 5 to the result of each solvent. The parallel circuit on the right in the red frame is configured to make the impedance of the SCL, and the middle part in the blue frame to make the impedance of the EDL. Using this equivalent circuit, the results of the impedance measurement were fit with the red lines in Figure A1, and the voltage distributed to the EDL and the electric field strength there were estimated at $210 \mathrm{~Hz}$. The impedance values for elements in the equivalent circuit are determined as given in Table A1. From the $\Delta n$ and $E$, the Pockels coefficients were derived from the following equation [13] as listed in Table 2. The accuracy of the coefficient values is estimated to be about $\pm 10 \%$.

$$
r_{13}\left[\frac{\mathrm{pm}}{\mathrm{V}}\right]=-\frac{2 \Delta n}{n^{3} E\left[\frac{\mathrm{v}}{\mathrm{m}}\right]} \times 10^{12}
$$

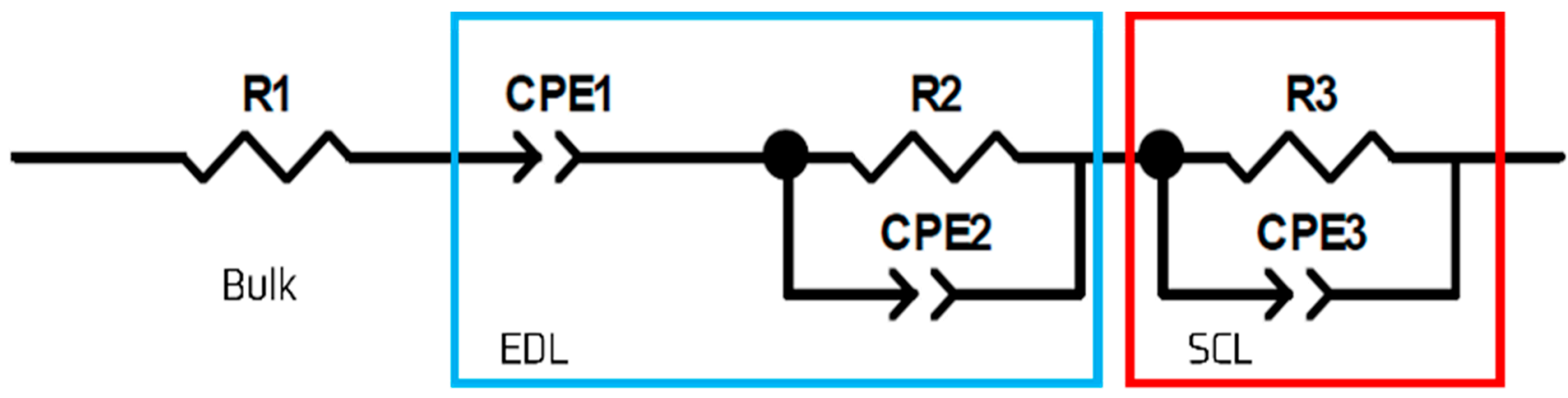

Figure 5. Equivalent circuit diagram with resistance $(\mathrm{R})$ and constant phase element (CPE) (see Appendix A).

Table 2. The Pockels coefficient determined for each solution in the EDL with the refractive index change $\Delta n$ and the electric field $E$ in the EDL when the thickness of the EDL is assumed to be $2 \mathrm{~nm}$.

\begin{tabular}{cccccc}
\hline Solvent & FA & NMF & DMF & [BMIM] [BF 4 & Water \\
\hline Relative permittivity & 109.5 & 186.0 & 37.4 & 13.9 & 80.2 \\
Density [g/cm ${ }^{3}$ ] & 1.133 & 1.011 & 0.944 & 1.21 & 0.998 \\
Viscosity [mPas] & 3.23 & 1.72 & 0.71 & 132 & 1.00 \\
Refractive index & 1.447 & 1.43 & 1.43 & 1.41 & 1.333 \\
Refractive index change & $-1.14 \times 10^{-2}$ & $-1.14 \times 10^{-2}$ & $-1.52 \times 10^{-2}$ & $-8.00 \times 10^{-3}$ & $-2.00 \times 10^{-2}$ \\
Intralayer electric field [V/m] & $9.11 \times 10^{7}$ & $9.74 \times 10^{7}$ & $8.66 \times 10^{7}$ & $1.05 \times 10^{8}$ & $1.02 \times 10^{8}$ \\
Pockels coefficient [pm/V] & 83 & 80 & 120 & 55 & 165 \\
\hline
\end{tabular}

\section{Discussion}

The Pockels coefficient of water is $165 \mathrm{pm} / \mathrm{V}$, which is smaller than the previously estimated values of about $200 \mathrm{pm} / \mathrm{V}$ [13] and $230 \mathrm{pm} / \mathrm{V}$ [16]. This can be attributed to the improvement in the impedance measurement and the equivalent circuit. In this paper, the electrodes were irradiated with white light during the impedance measurement as in the electric-field modulation experiments (no irradiation in Refs. $[13,16])$. As the equivalent 
circuit, a constant phase element (CPE) was used instead of capacitance (capacitance used in Ref. [12]), and the measurement frequency range was widened compared to Refs. [13,16]. Furthermore, the addition of CPE in series to represent the EDL as in Figure 5 has improved the fit in the low-frequency range (the line running diagonally up in the Cole-Cole plot). As a result, while the fit was not perfect in Refs. [13,16], an almost perfect fit was obtained in the present study, resulting in a more accurate estimate of the electric field in the layer.

The estimated Pockels coefficients of FA, NMF, and DMF are 83, 80, and $120 \mathrm{pm} / \mathrm{V}$, respectively, all of which are lower than the $165 \mathrm{pm} / \mathrm{V}$ of water. The Pockels coefficient of IL is $55 \mathrm{pm} / \mathrm{V}$, which is also not higher than that of water. From the Pockels coefficient of the three amides, one can say that the static dielectric constant of the solvent is not an important factor in determining the magnitude of the Pockels coefficient.

It is also interesting to note that the $\Delta T / T$ spectrum of IL is nearly the same in shape as that of water despite the fact that they are completely different types of liquids, considering the following differences. In case we regard the ionic liquid as an $100 \%$ electrolyte solution, the molar concentration of the electrolyte in [BMIM] $\left[\mathrm{BF}_{4}\right]$ is calculated to be $5.3 \mathrm{M}$ (density $1.2011[\mathrm{~g} / \mathrm{mL}] \times 1000[\mathrm{~mL} / \mathrm{L}] /$ molecular weight $226.03=5.3139$ ), but as shown in Appendix $\mathrm{A}$, the impedance measurements show that the resistance of the bulk liquid part is not much different from other liquids of $0.1 \mathrm{M}$ solution. On the other hand, the thickness of the EDL is on the order of $0.1 \mathrm{~nm}$ if the Debye length is applied as it is [8], which is $1 / 10$ of the size of the constituent ions of IL, so it is obvious that the Debye length cannot be applied as it is, and the structure of the EDL is still one of the controversial topics [8-10].

It has been previously reported [16] that $\mathrm{MeOH}$ and $\mathrm{EtOH}$, which are both polar protic solvents with relatively strong hydrogen-bonding, have relatively large Pockels coefficients, although they are smaller than that of water. In that paper, it was concluded that the Pockels coefficient of water, $\mathrm{MeOH}, \mathrm{EtOH}$, and DMSO at the interface of transparent electrode scales with (hydrogen-bond strength)/(viscosity). FA and NMF are polar protic solvents, and it is known that strong hydrogen bonding is the reason for their large static dielectric constants [23]. On the other hand, DMF is a polar aprotic solvent similar to DMSO. From Ref. [16], it is, therefore, expected that FA and NMF have larger Pockels coefficient than DMF, but the present results show that the Pockels coefficient is not necessarily scaled by (hydrogen-bond strength)/(viscosity).

Combining the present results with the previously reported results, it is most likely that water has the largest Pockels coefficient (which should be called the interfacial Pockels coefficient) among all liquids. We will discuss possible reasons for this from a different perspective. It has been discussed that the Pockels effect in bulk solid crystals is due to the constituent ions displaced from their symmetric positions, which is the element that breaks the inversion symmetry: When an electric field is applied, the ions either move further away from or closer to the symmetry position, resulting in the field-direction dependent change in the electronic distribution through electron-phonon coupling [15]. The change in the electronic state is required for the Pockels effect because it is the change in the refractive index in the visible region. Since the refractive index is a quantity that reflects the magnitude of the polarization response in phase with the optical electric field at that frequency, it is caused by the oscillator, which can respond without delay to the visible optical field, consisting of electronic transitions whose resonant frequency is greater than the visible frequency. Therefore, the Pockels effect of water at the electrode interface may also be caused by "changes in the electronic state of water" brought about by the electricfield induced orientation, deformation, density change of water molecules, interaction with the electrode, biased distribution of electrolyte ions, etc. The refractive index of water is 1.33 , the smallest of almost all liquids at room-temperature. The reason for this is not the density (which is not so small as most organic solvents), but the fact that the electronic transitions are at the shortest wavelength (start to rise at a wavelength around $170 \mathrm{~nm}$ ). (Note that $\mathrm{MeOH}$ has a refractive index of 1.329, smaller than water. The mass density of $\mathrm{MeOH}$ is $80 \%$ of that of water, so it should be even smaller than 1.329 , but it is rather closer 
to the value for water, indicating that the electronic transitions are at longer wavelengths than water. In fact, however, far-UV absorbances of alkane, alcohol, and water similarly start to rise around $170 \mathrm{~nm}$ [34], so that full explanation of their refractive indices in terms of density and electronic transition is not obvious).

From this point of view, the refractive index change due to the structural change should be larger in solvents with lower electronic transition energy than water (This should be true for FA, NMF, DMF, DMSO, and IL $[35,36]$ with refractive indices larger than water), but this is not the case, i.e., the Pockels coefficients of these liquids are smaller than that of water. This suggests that the change in the electronic transitions caused by the electric-field induced structural change is the largest (i.e., structural change or electron-phonon coupling is the largest) in water among all solvents and liquids.

\section{Conclusions and Prospect}

The Pockels coefficients of various liquids in the EDL formed at the interface between the transparent oxide electrode and the solution were measured. The coefficients were found to be in the following order in magnitude: water, DMF, FA, NMF, and imidazolium IL. When combined with the previous results for alcohols and DMSO, it is most likely that water has the largest Pockels coefficient among all room-temperature liquids. The strength of hydrogen bond and the magnitude of the static dielectric constant do not seem to be decisive factors in determining the magnitude of the Pockels coefficient for the liquid-electrode interfacial Pockels effect.

Although this is a hypothesis, the fact that water has the largest Pockels coefficient despite its small refractive index may indicate that water has a large change in electronic state due to structural deformation caused by the application of an electric field, since the density and electronic transition energy are the major factors controlling the size of the refractive index of visible light in transparent materials. Therefore, research focusing on the electronic spectrum of water is also desirable to elucidate the mechanism.

Author Contributions: Conceptualization, A.O. and E.T.; methodology, A.O. and E.T.; software, A.O. and E.T.; validation, A.O. and E.T.; formal analysis, A.O. and E.T.; investigation, A.O.; resources, E.T. and T.K.; data curation, A.O.; writing-original draft preparation, A.O. and E.T.; writing-review and editing, E.T. and T.K.; visualization, A.O.; supervision, E.T.; project administration, E.T.; funding acquisition, E.T. All authors have read and agreed to the published version of the manuscript.

Funding: This research was funded by Grant in Aid for Scientific Research(B) (Grant Number JP20 H02659), Japan Society for the Promotion of Science (JSPS).

Data Availability Statement: The data that support the findings of this study are available from the corresponding author upon reasonable request.

Conflicts of Interest: The authors declare no conflict of interest.

\section{Appendix A}

On the equivalent circuit for fitting the results of impedance measurement, each element of the equivalent circuit and its determined value is listed for all liquids in Table A1 and the results of the measurement and the fitting curves with these values are shown in Figure A1.

$\mathrm{R}$ : Resistance, impedance is $Z_{R}=r$.

CPE: pseudo-capacitance (constant phase element), impedance is $Z_{C P E}=\frac{1}{T(j \omega)^{p}}$ $(0<p<1, \omega:$ angular frequency). 

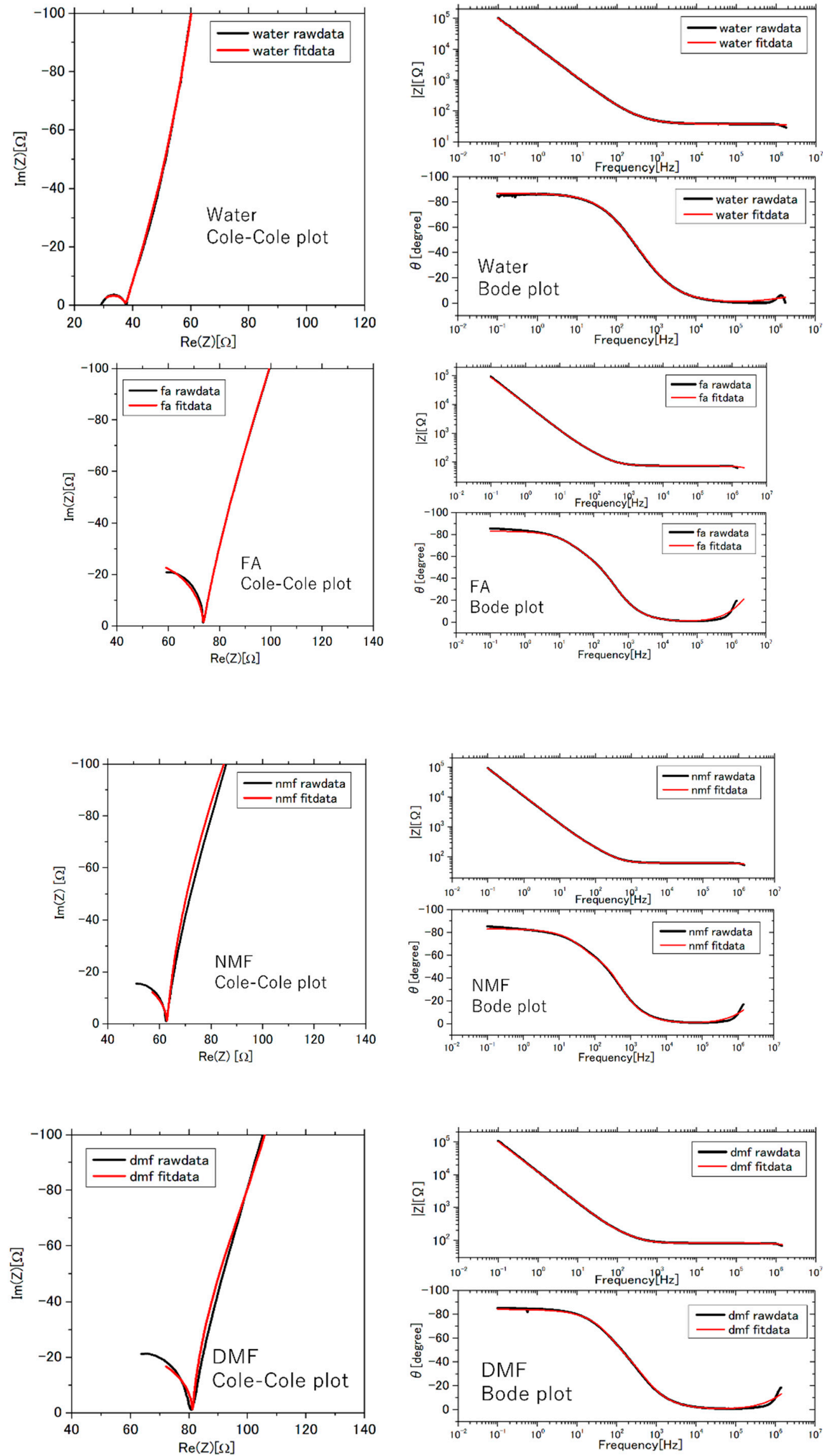

Figure A1. Cont. 

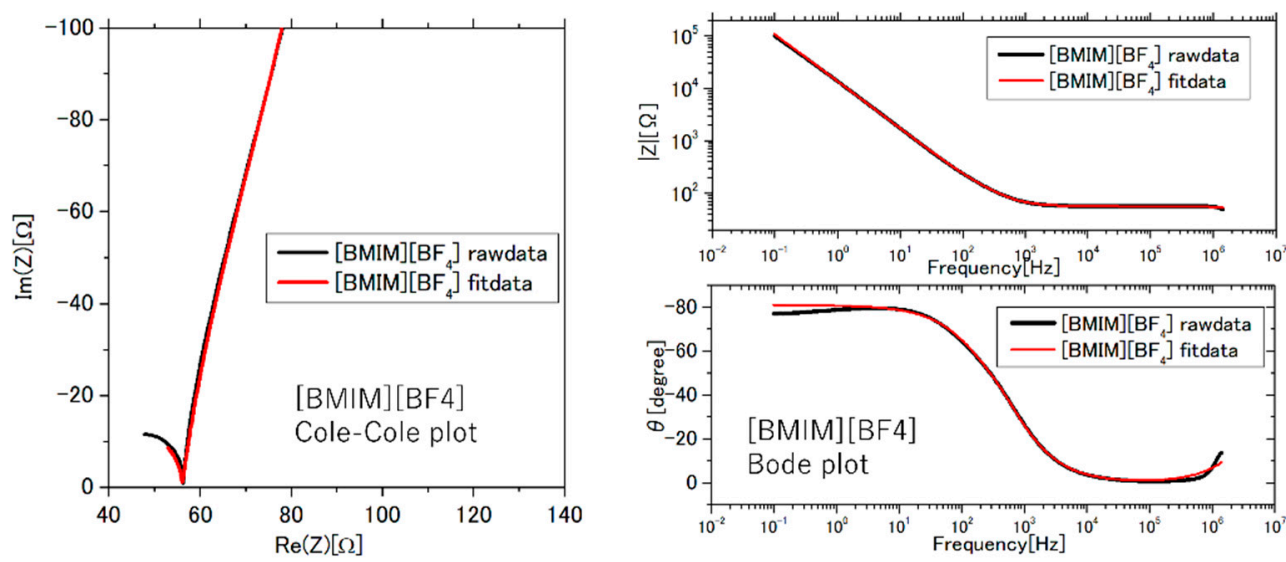

Figure A1. The results of impedance measurements (black lines) and the fitting curves (red lines).

Table A1. Fitting parameters of each solvent and fitted values for them.

\begin{tabular}{|c|c|c|c|c|}
\hline Water & FA & DMF & NMF & {$[\mathrm{BMIM}]\left[\mathrm{BF}_{4}\right]$} \\
\hline \multicolumn{5}{|c|}{ Bulk } \\
\hline $\mathrm{r} 1=29.16$ & $\mathrm{r} 1=23.85$ & $\mathrm{r} 1=41.28$ & $\mathrm{r} 1=31.04$ & $\mathrm{r} 1=28.17$ \\
\hline \multicolumn{5}{|c|}{ EDL } \\
\hline $\mathrm{CPE}-\mathrm{t} 1=1.5571 \times 10^{-5}$ & $\mathrm{CPE}-\mathrm{t} 1=1.7448 \times 10^{-5}$ & $\mathrm{CPE}-\mathrm{t} 1=1.5076 \times 10^{-5}$ & CPE-t1 $=1.6946 \times 10^{-5}$ & CPE-t $1=1.4051 \times 10^{-5}$ \\
\hline CPE-p1 $=0.96306$ & CPE-p1 = 0.92312 & CPE-p1 $=0.93415$ & CPE-p1 $=0.92243$ & CPE-p1 $=0.89908$ \\
\hline $\mathrm{r} 2=8.349$ & r2 $=49.68$ & r2 = 39.91 & $\mathrm{r} 2=31.77$ & r2 $=27.78$ \\
\hline CPE-t2 $=8.92 \times 10^{-8}$ & $\begin{array}{c}\text { CPE-t } 2=9.1887 \times \\
10^{-10}\end{array}$ & $\mathrm{CPE}-\mathrm{t} 2=1.5238 \times 10^{-8}$ & CPE-t2 $=1.6568 \times 10^{-9}$ & CPE-t2 $=1.409 \times 10^{-9}$ \\
\hline CPE-p2 = 0.83177 & CPE-p2 = 1 & CPE-p2 = 1 & CPE-p2 = 1 & CPE-p2 = 1 \\
\hline \multicolumn{5}{|c|}{ SCL } \\
\hline $\mathrm{r} 3=32.75$ & $\mathrm{r} 3=82.61$ & r3 $=29.49$ & $\mathrm{r} 3=51.14$ & $\mathrm{r} 3=12.59$ \\
\hline CPE-t3 $=0.00023749$ & CPE-t3 $=7.4832 \times 10^{-5}$ & $\mathrm{CPE}-\mathrm{t} 3=2.5267 \times 10^{-5}$ & $\mathrm{CPE}-\mathrm{t} 3=2.6216 \times 10^{-5}$ & CPE-t3 $=4.4851 \times 10^{-5}$ \\
\hline CPE-p3 = 0.69759 & CPE-p3 = 0.83291 & CPE-p3 = 1 & CPE-p3 = 1 & CPE-p3 = 1 \\
\hline
\end{tabular}

\section{References}

1. Schmickler, W. Electronic effects in the electric double layer. Chem. Rev. 1996, 96, 3177-3200. [CrossRef] [PubMed]

2. Brown, G.E.; Henrich, V.E.; Casey, W.H.; Clark, D.L.; Eggleston, C.; Felmy, A.; Goodman, D.W.; Grätzel, M.; Maciel, G.; McCarthy, M.I.; et al. Metal oxide surfaces and their interactions with aqueous solutions and microbial organisms. Chem. Rev. 1999, 99, 77-174. [CrossRef] [PubMed]

3. Burt, R.; Birkett, G.; Zhao, X.S. A review of molecular modelling of electric double layer capacitors. Phys. Chem. Chem. Phys. 2014, 16, 6519-6538. [CrossRef]

4. Shimotani, H.; Asanuma, H.; Tsukazaki, A.; Ohtomo, A.; Kawasaki, M.; Iwasa, Y. Insulator-to-metal transition in ZnO by electric double layer gating. Appl. Phys. Lett. 2007, 91, 082106. [CrossRef]

5. Scherwitzl, R.; Zubko, P.; Lezama, I.G.; Ono, S.; Morpurgo, A.F.; Catalan, G.; Triscone, J.-M. Electric-field control of the metalinsulator transition in ultrathin NdNiO3 films. Adv. Mater. 2010, 22, 5517-5520. [CrossRef] [PubMed]

6. Electrically Induced Ferromagnetism at Room Temperature in Cobalt-Doped Titanium Dioxide IScience. Available online: https: / / science.sciencemag.org/content/332/6033/1065 (accessed on 22 June 2021).

7. Ueno, K.; Nakamura, S.; Shimotani, H.; Ohtomo, A.; Kimura, N.; Nojima, T.; Aoki, H.; Iwasa, Y.; Kawasaki, M. Electric-fieldinduced superconductivity in an insulator. Nat. Mater. 2008, 7, 855-858. [CrossRef]

8. Bazant, M.Z.; Storey, B.D.; Kornyshev, A.A. Double layer in ionic liquids: Overscreening versus crowding. Phys. Rev. Lett. 2011, 106, 046102. [CrossRef] [PubMed]

9. Yamamoto, R.; Morisaki, H.; Sakata, O.; Shimotani, H.; Yuan, H.; Iwasa, Y.; Kimura, T.; Wakabayashi, Y. External electric field dependence of the structure of the electric double layer at an ionic liquid/Au interface. Appl. Phys. Lett. 2012, 101, 053122. [CrossRef] 
10. Fedorov, M.V.; Kornyshev, A.A. Ionic liquids at electrified interfaces. Chem. Rev. 2014, 114, 2978-3036. [CrossRef]

11. Zhao, Z.; Zhang, G.; Yin, Y.; Dong, C.; Liu, Y.D. The electric field responses of inorganic ionogels and poly(ionic liquid)s. Molecules 2020, 25, 4547. [CrossRef]

12. Tokunaga, E.; Nosaka, Y.; Hirabayashi, M.; Kobayashi, T. Pockels effect of water in the electric double layer at the interface between water and transparent electrode. Surf. Sci. 2007, 601, 735-741. [CrossRef]

13. Nosaka, Y.; Hirabayashi, M.; Kobayashi, T.; Tokunaga, E. Gigantic optical pockels effect in water within the electric double layer at the electrode-solution interface. Phys. Rev. B 2008, 77, 241401. [CrossRef]

14. Weis, R.S.; Gaylord, T.K. Lithium niobate: Summary of physical properties and crystal structure. Appl. Phys. A 1985, 37, 191-203. [CrossRef]

15. Meng, X.Y.; Wang, Z.Z.; Zhu, Y.; Chen, C.T. Mechanism of the electro-optic effect in the perovskite-type ferroelectric $\mathrm{KNbO}_{3}$ and $\mathrm{LiNbO}_{3}$. J. Appl. Phys. 2007, 101, 103506. [CrossRef]

16. Kanemaru, H.; Yukita, S.; Namiki, H.; Nosaka, Y.; Kobayashi, T.; Tokunaga, E. Giant pockels effect of polar organic solvents and water in the electric double layer on a transparent electrode. RSC Adv. 2017, 7, 45682-45690. [CrossRef]

17. Yukita, S.; Suzuki, Y.; Shiokawa, N.; Kobayashi, T.; Tokunaga, E. Mechanisms of the anomalous pockels effect in bulk water. Opt. Rev. 2018, 25, 205-214. [CrossRef]

18. Nishi, Y.; Watanabe, R.; Sasaki, S.; Okada, A.; Seto, K.; Kobayashi, T.; Tokunaga, E. Electric-field induced shift in the plasmon resonance due to the interfacial pockels effect of water on a silver surface. Appl. Sci. 2021, 11, 2152. [CrossRef]

19. Hayama, D.; Seto, K.; Yamashita, K.; Yukita, S.; Kobayashi, T.; Kobayashi, T.; Kobayashi, T.; Tokunaga, E.; Tokunaga, E. Giant pockels effect in an electrode-water interface for a "liquid" light modulator. OSA Contin. 2019, 2, 3358-3373. [CrossRef]

20. Teng, P.; Luo, M.; Yang, X.; Gao, D.; Copner, N.; Liu, Z.; Liu, Z.; Yang, J.; Li, Z.; Gao, S.; et al. All-fiber bidirectional optical modulator derives from the microfiber coated with ITO electrode. Opt. Lett. 2021, 46, 2497-2500. [CrossRef]

21. Kanemaru, H.; Nosaka, Y.; Hirako, A.; Ohkawa, K.; Kobayashi, T.; Tokunaga, E. Electrooptic effect of water in electric double layer at interface of GaN electrode. Opt. Rev. 2010, 17, 352-356. [CrossRef]

22. Puranik, S.M.; Kumbharkhane, A.C.; Mehrotra, S.C. Dielectric study of formamide, N-methyl-formamide and N, Ndimethylformamide. Indian J. Chem.-Sect. A 1993, 32, 613-615.

23. Richardi, J.; Krienke, H.; Fries, P.H. Dielectric constants of liquid formamide, N-methylformamide and dimethylformamide via molecular ornstein-zernike theory. Chem. Phys. Lett. 1997, 273, 115-121. [CrossRef]

24. Deetlefs, M.; Seddon, K.R.; Shara, M. Predicting physical properties of ionic liquids. Phys. Chem. Chem. Phys. 2006, 8, 642-649. [CrossRef] [PubMed]

25. Tomida, D.; Kumagai, A.; Qiao, K.; Yokoyama, C. Viscosity of [Bmim][PF6] and [Bmim][BF 4 at high pressure. Int. J. Thermophys. 2006, 1, 39-47. [CrossRef]

26. Ludwig, R. Water: From clusters to the bulk. Angew. Chem. Int. Ed. 2001, 40, 1808-1827. [CrossRef]

27. Neumann, M. Dielectric relaxation in water. Computer simulations with the TIP4P potential. J. Chem. Phys. 1986, 85, 1567-1580. [CrossRef]

28. Toda, S.; Clark, R.; Welton, T.; Shigeto, S. Observation of the pockels effect in ionic liquids and insights into the length scale of potential-induced ordering. Langmuir 2021, 37, 5193-5201. [CrossRef]

29. Huang, M.-M.; Jiang, Y.; Sasisanker, P.; Driver, G.W.; Weingärtner, H. Static relative dielectric permittivities of ionic liquids at $25^{\circ}$ C. J. Chem. Eng. Data 2011, 56, 1494-1499. [CrossRef]

30. Born, M.; Wolf, E. Principles of Optics: 60th Anniversary Edition, 7th ed.; Cambridge University Press: Cambridge, UK, 2019; ISBN 978-1-108-47743-7.

31. Hamberg, I.; Granqvist, C.G.; Berggren, K.-F.; Sernelius, B.E.; Engström, L. Band-gap widening in heavily Sn-doped In $2 \mathrm{O}_{3}$. Phys. Rev. B 1984, 30, 3240-3249. [CrossRef]

32. Glosser, R.; Seraphin, B.O. Band-population effects in the electroreflectance spectrum of InSb. Phys. Rev. 1969, 187, 1021-1024. [CrossRef]

33. Bottka, N.; Johnson, D.L.; Glosser, R. Band-population interference phenomena in the electroreflectance of narrow-gap semiconductors under heavy surface accumulation. Phys. Rev. B 1977, 15, 2184-2194. [CrossRef]

34. Ozaki, Y.; Morisawa, Y.; Ikehata, A.; Higashi, N. Far-ultraviolet spectroscopy in the solid and liquid states: A review. Appl. Spectrosc. 2012, 66, 1-25. [CrossRef]

35. Morisawa, Y.; Yasunaga, M.; Fukuda, R.; Ehara, M.; Ozaki, Y. Electronic transitions in liquid amides studied by using attenuated total reflection far-ultraviolet spectroscopy and quantum chemical calculations. J. Chem. Phys. 2013, 139, 154301. [CrossRef] [PubMed]

36. Ozaki, Y.; Morisawa, Y.; Tanabe, I.; Beć, K.B. ATR-far-ultraviolet spectroscopy in the condensed phase-The present status and future perspectives. Spectrochim. Acta Part A Mol. Biomol. Spectrosc. 2021, 253, 119549. [CrossRef] [PubMed] 